\title{
A Robust Motion Artifact Detection Algorithm for Accurate Detection of Heart Rates From Photoplethysmographic Signals Using Time-Frequency Spectral Features
}

\author{
Duy Dao, S. M. A. Salehizadeh, Yeonsik Noh, Jo Woon Chong, Chae Ho Cho, Dave McManus, \\ Chad E. Darling, Yitzhak Mendelson, and Ki H. Chon, Senior Member, IEEE
}

\begin{abstract}
Motion and noise artifacts (MNAs) impose limits on the usability of the photoplethysmogram (PPG), particularly in the context of ambulatory monitoring. MNAs can distort PPG, causing erroneous estimation of physiological parameters such as heart rate (HR) and arterial oxygen saturation (SpO2). In this study, we present a novel approach, "TifMA," based on using the time-frequency spectrum of PPG to first detect the MNA-corrupted data and next discard the nonusable part of the corrupted data. The term "nonusable" refers to segments of PPG data from which the HR signal cannot be recovered accurately. Two sequential classification procedures were included in the TifMA algorithm. The first classifier distinguishes between MNA-corrupted and MNA-free PPG data. Once a segment of data is deemed MNA-corrupted, the next classifier determines whether the HR can be recovered from the corrupted segment or not. A support vector machine (SVM) classifier was used to build a decision boundary for the first classification task using data segments from a training dataset. Features from timefrequency spectra of PPG were extracted to build the detection model. Five datasets were considered for evaluating TifMA performance: (1) and (2) were laboratory-controlled PPG recordings from forehead and finger pulse oximeter sensors with subjects making random movements, (3) and (4) were actual patient PPG recordings from UMass Memorial Medical Center with random free movements and (5) was a laboratory-controlled PPG recording dataset measured at the forehead while the subjects ran on a treadmill. The first dataset was used to analyze the noise sensitivity of the algorithm. Datasets 2-4 were used to evaluate the MNA detection phase of the algorithm. The results from the first phase
\end{abstract}

Manuscript received April 26, 2016; revised August 16, 2016; accepted September 18, 2016. Date of publication October 21, 2016; date of current version September 1, 2017. This work was supported in part by the US Army Medical Research and Materiel Command under Grant W81XWH-12-1-0541 and the NSF award \#1522087.

D. Dao, S. M. A. Salehizadeh, Y. Noh, and K. H. Chon are with the University of Connecticut, Storrs, CT 06269 USA (e-mail: dkdao2013@gmail.com; smasalehizadeh@gmail.com; ysnoh@engr. uconn.edu; ki.chon@uconn.edu).

J. W. Chong and C. H. Cho are with Texas Tech University, Lubbock, TX 79409 USA (e-mail: jw9607@gmail.com; chae.cho@uconn.edu).

D. McManus and C. E. Darling are with the University of Massachusetts Medical School, Worcester, MA 01655 USA (e-mail: mcmanus.dave@gmail.com; Chad.Darling@umassmed.edu).

Y. Mendelson is with Worcester Polytechnic Institute, Worcester, MA 01609 USA (e-mail: ym @ wpi.edu).

Digital Object Identifier 10.1109/JBHI.2016.2612059 of the algorithm (MNA detection) were compared to results from three existing MNA detection algorithms: the Hjorth, kurtosis-Shannon entropy, and time-domain variability-SVM approaches. This last is an approach recently developed in our laboratory. The proposed TifMA algorithm consistently provided higher detection rates than the other three methods, with accuracies greater than $95 \%$ for all data. Moreover, our algorithm was able to pinpoint the start and end times of the MNA with an error of less than $1 \mathrm{~s}$ in duration, whereas the next-best algorithm had a detection error of more than $2.2 \mathrm{~s}$. The final, most challenging, dataset was collected to verify the performance of the algorithm in discriminating between corrupted data that were usable for accurate HR estimations and data that were nonusable. It was found that on average $48 \%$ of the data segments were found to have MNA, and of these, $38 \%$ could be used to provide reliable HR estimation.

Index Terms-Complex demodulation (CDM), heart rate (HR) estimation, motion and noise artifacts (MNAs), photoplethysmography, time-frequency (TF).

\section{INTRODUCTION}

$\mathbf{P}$ ULSE oximeter (PO) is a noninvasive, low-cost device that is widely used in hospitals and clinics to monitor heart rate (HR) and arterial oxygen saturation (SpO2). Recently, there have been efforts to derive other physiological parameters from photoplethysmogram (PPG), as recorded by a PO [1]-[3]. The fluctuations observed in a PPG are influenced by arterial and venous blood flow, as well as the autonomic and respiratory systems of the peripheral circulation. Such information could be used more comprehensively for phenotyping cardiovascular health. Due to increasing health care costs, a single sensor from which multiple clinical data points can be derived such as a PO is very attractive from a financial perspective. Moreover, using a PO as a multipurpose vital sign monitor has clinical appeal, since the device is widely accepted by clinicians and patients because of its ease of use, comfort, and accuracy in providing reliable vital signs. Knowledge of respiratory rate and HR patterns would provide useful clinical information in many situations where a PO is the sole available monitor. However, extraction of vital signs and other physiological parameters using pulse oximetry is predicated on motion artifact-free PPG data. It is well known 
that the PPG is highly sensitive to artifacts, particularly those generated while the patient is in motion [4]. This imposes a huge limitation on the usability of the PPG for ambulatory monitoring applications. Motion and noise artifacts (MNAs) distorting PPG recordings can cause erroneous estimation of $\mathrm{HR}$ and $\mathrm{SpO} 2$ [5]. Although the intelligent design of sensor attachment, form factors, and packaging can help to reduce the impact of motion disturbances by making sure that the sensor is securely mounted, they are not sufficient for complete MNA removal. Combating MNAs in PPG data has been the core focus of research for many years.

Although there are techniques which have been proposed to alleviate the effects of MNAs, the solution to this problem still remains unsatisfactory in practice. Several algorithm-based MNA reduction methods were proposed, such as time- and frequency-domain filtering, power spectrum analysis, and blind source separation techniques [6]-[12]. These techniques reconstruct noise-contaminated PPG data so that a noise-reduced signal is obtained. However, the reconstructed signal typically contains incomplete dynamic features of the uncorrupted PPG signal, and some algorithms are solely designed to capture only the HR and $\mathrm{SpO} 2$ information instead of the signal's morphology and its amplitudes, which are needed for other physiological derivations [13]. Moreover, these reconstruction algorithms operate even on clean PPG portions where MNA reduction is not needed. This introduces unnecessary computation burdens and distorts the signal integrity of the clean portion of the data. Hence, an accurate MNA detection algorithm, which distinguishes clean PPG recording portions from corrupted portions, is essential to not distort the noncorrupted data segments [14].

MNA detection methods are mostly based on a signal quality index (SQI) which quantifies the severity of the artifacts. Some approaches calculate the SQI using waveform morphologies [15]-[17] or filtered output [18], [19], while others derive the SQI with the help of additional hardware such as an accelerometer and electrocardiogram (ECG) [20], [21]. In some commercially available POs, accelerometers are not provided, or even if they are available, access to the raw data is not usually feasible; hence, they cannot be used for MNA cancellation. Moreover, traditional approaches to cancellation of MNA using adaptive noise filtering do not always yield accurate results.

Statistical measures, such as skewness, kurtosis (K), and quadratic phase coupling [22], Shannon entropy (SE), and Renyi's entropy [23], have been shown to be helpful in determining the SQI. These statistical algorithms discriminate amplitude distributions between PPG segments with an assumption that clean and corrupt segments would form two separate groups. However, PPG morphology varies among patients, thus yielding a multitude of amplitude distributions. Therefore, it would be difficult to obtain high accuracy from these algorithms in practice. Another approach, using Hjorth parameters, where H1 and $\mathrm{H} 2$ represent central frequency and half of the bandwidth of a signal, respectively, were proposed as means to quantify the degree of oscillation in a signal [24], [25]. They were employed for MNA discrimination in PPG by Gil et al. [26] with a hypothesis that MNA-derived $\mathrm{H} 1$ and $\mathrm{H} 2$ would largely differ from $\mathrm{H} 1$ and $\mathrm{H} 2$ derived from the noise-free PPG signal. However, due to the time-varying dynamic nature of PPG signals, fre- quency features such as $\mathrm{H} 1$ and $\mathrm{H} 2$ alone would not be sufficient for accurate detection of MNA. Our recently published MNA detection method for PPG uses time-domain features such as variability in $\mathrm{HR}$, amplitude, and waveform morphology, with the help of the support vector machine (SVM) classifier, for detection [27]. The algorithm, which we termed time-domain variability (TDV) SVM, was shown to be more robust than other statistical-based algorithms as it uses successive difference and variability measures. However, this method is highly dependent on accuracy of the peak amplitude detection. Unlike the ECG, the PPG waveform does not have distinctive peaks which make accurate peak detection challenging. The dependence on a peak detection subroutine is a drawback of the TDV algorithm and inevitably affects its performance.

Time-frequency (TF) techniques such as smoothed pseudo Wigner-Ville, short-time Fourier transform, continuous wavelet transform, Hilbert-Huang transform, and variable frequency complex demodulation (VFCDM) received considerable attention as a means to analyze the signal of interest in both temporal and spectral domains [28]-[30]. Yan et al. used a smoothed pseudo Wigner-Ville TF technique for MNA reduction albeit with limited success [10]. In this paper, we introduce a novel algorithm for MNA detection using a TF representation produced by VFCDM. It is hypothesized in the design of our proposed MNA detection algorithm that TF information would provide meaningful dynamic features for improved differentiation of MNAs.

In this paper, we present a new MNA detection algorithm, "TifMA," which not only detects MNA-corrupted PPG segments but also is able to discriminate between usable versus nonusable PPG segments. The TifMA algorithm was developed based on the features from the time-frequency spectrum (TFS) of a PPG signal. The variable frequency demodulation technique was used to derive the TFS of PPG. The proposed algorithm includes two phases: 1) MNA detection; and 2) deciding whether or not an MNA-corrupted data segment can be used. The algorithm performance was evaluated at each phase using different PPG recordings. We show that features from TF spectra of PPG have good accuracy in discriminating between MNA-corrupted and clean PPG data; in addition, the algorithm can provide an ability to determine if the corrupted data are usable or not for estimating HR. In this work, features that are extracted from VFCDM TF were used as inputs for the machine-learning classifier algorithm which utilized the SVM. The results of the MNA detection phase of TifMA were compared to three other existing MNA detection algorithms: the Hjorth [26], kurtosis-SE [23], and the TDV-SVM approach, an approach recently developed in our laboratory [27]. The output of the usability detection stage of the algorithm was obtained according to a fixed threshold value and was evaluated by comparing the reference HR to the estimated HR from a VFCDM TFS.

\section{MATERIALS AND EXPERIMENTS}

\section{A. Experimental Protocol and Preprocessing}

To develop, analyze, and evaluate the proposed TifMA algorithm, we used five different datasets. Datasets (1) and (2) were recorded in controlled conditions from ten subjects in the Chon 
TABLE I

PPG DATASETS AND EXPERIMENTS SETTINGS

\begin{tabular}{lccc}
\hline \hline No. subjects & Dataset & Electrode type & Subject's age/sex \\
\hline 11 & 1 (Chon Lab) & Forehead PO & $23-58$ y (10 male, 1 female) \\
11 & 2 (Chon Lab) & Finger PO & $23-58$ y (10 male, 1 female) \\
10 & 3 (UMMC) & Forehead PO & $18-38$ y (5 female, 5 male) \\
10 & 4 (UMMC) & Finger PO & $18-45$ y (4 female, 6 male) \\
10 & 5 (Chon Lab) & Forehead PO & $23-58$ y (9 male, 1 female) \\
\hline \hline
\end{tabular}

Lab and datasets (3) and (4) were provided by UMASS Hospital, recorded from ten patients. Dataset (5) was also recorded in the Chon Lab, but during a treadmill experiment. This study was approved by both Worcester Polytechnic Institute's (WPI) and UMass Memorial Medical Center's (UMMC) IRBs and all subjects provided informed consent prior to data recording. All laboratory recordings were performed in a quiet room with a temperature of $70^{\circ} \mathrm{F}$. There are two versions with different form factors to capture PPG at the user's forehead and finger. The forehead sensor is termed 6PD-forehead since it consists of six photodetectors concentric around the center-paired LEDs, which have peak wavelengths of $660 \mathrm{~nm}$ (red) and $940 \mathrm{~nm}$ (infrared). The finger sensor, due to space constraints, consists of three photodetectors concentric around center-paired LEDs with the same specifications as those of the 6PD-forehead sensor. The PPGs were sampled at $80 \mathrm{~Hz}$. The scope of this study was to evaluate the efficacy of our proposed TifMA algorithm. Therefore, only one infrared PPG channel, deemed to contain the best signal quality data, was used for analysis. Details of this study's database including demographics of subjects are given in Table I.

The details of experiments and data collection protocols are as follows.

1) Datasets: (1) and (2) Chon Lab dataset: For the laboratory-controlled environment, both forehead and finger worn PO sensor data were collected from healthy subjects recruited from the student community of WPI. Laboratory data allow us to have more control over the duration of the MNA generated, to ensure that the detection phase of the algorithm was tested on a wide range of MNA durations. In laboratorycontrolled PPG data, motion artifacts were induced by head and finger movements for specific time intervals in both horizontal and vertical directions. For head movement data, 11 volunteers were asked to wear our PO on their foreheads along with a reference Masimo Radical (Masimo SET) finger type transmittance PO. The subjects were all healthy with no past histories of cardiovascular disease. After baseline recording for $5 \mathrm{~min}$ without any movement, subjects were instructed to introduce motion artifacts for specific time intervals varying from $10 \%$ to $50 \%$ within a 1 min segment. For example, if a subject was instructed to perform left-right random movements for $6 \mathrm{~s}$, a 1 min segment of data would contain $10 \%$ noise. The laboratory finger movement data were recorded in a similar way as the head data using our custom-made PPG finger sensor on the moving finger and the reference Masimo PO on the stationary hand.
TABLE II

Averaged Cohen's $\kappa$ Coefficients Representing the AgReEment BETWEEN INSPECTORS' DECISIONS ON PPG LABELS

\begin{tabular}{lcccc}
\hline \hline & Lab. head & Lab. finger & UMass. head & UMass. finger \\
\hline Cohan's $\kappa$ & 0.823 & 0.935 & 0.732 & 0.791 \\
\# of subjects & 11 & 11 & 10 & 10 \\
Duration (min) & 5 & 5 & 10 & 10 \\
\hline \hline
\end{tabular}

TABLE III

Mean \pm Std. Deviation of Performance Metrics of Our Proposed TIFMA USING VARIOUS WINDOW LENGTHS

\begin{tabular}{cccccc}
\hline \hline & $\mathrm{L}_{2}$ & $3 \mathrm{~s}$ & $4 \mathrm{~s}$ & $5 \mathrm{~s}$ & $6 \mathrm{~s}$ \\
\hline Lab. head & Accuracy & $86.8 \pm 5.52$ & $95.7 \pm 0.82$ & $86.9 \pm 4.27$ & $85.5 \pm 6.96$ \\
& Sensitivity & $82.0 \pm 9.59$ & $93.0 \pm 5.75$ & $81.5 \pm 8.47$ & $79.8 \pm 9.58$ \\
& Specificity & $89.1 \pm 5.86$ & $96.6 \pm 1.48$ & $81.5 \pm 3.19$ & $90.0 \pm 5.97$ \\
\hline \hline
\end{tabular}

(3) and (4) UMMC dataset: The next dataset was acquired from patients who were admitted to our partner hospital at the UMMC. Data from patients provided the most realistic information on the motion artifacts since the patients were allowed to move freely as long as the sensors were positioned properly. The patient PPG data were recorded from ten subjects admitted to emergency department at UMMC. Similar to the laboratory-controlled dataset, each patient was fitted with our custom-made sensors (both forehead and finger) and the Masimo POs on the finger of opposite hand. One subject had hypertension and two subjects suffered from hypercholesterolemia and hyperlipidemia; the remaining subjects were considered free from cardiovascular diseases. All recording were performed in the Emergency Department with a room temperature of $68^{\circ} \mathrm{F}$. The patients were admitted due to pain symptoms and were not restrained from making natural movements. Therefore, they would have generated many different but natural characteristics of MNA in their recorded PPG data.

(5) Chon Lab dataset: This dataset was also recorded in the Chon Lab from ten healthy subjects, with ages ranging from 26 to 55. For each subject, the PPG signal was recorded from their forehead using a PO. The lead II ECG signal was recorded as a reference from the chest using ECG sensors, sampled at $400 \mathrm{~Hz}$. During data recording, subjects walked, jogged, and ran on a treadmill with speeds of 3,5 , and $7 \mathrm{~m} / \mathrm{h}$, respectively, for $9 \mathrm{~min}$. At the end, all experimental subjects were asked to perform random arbitrary movements. The activity durations were $1 \mathrm{~min}$ rest, $1 \mathrm{~min}$ walking $(3 \mathrm{~m} / \mathrm{h}), 1 \mathrm{~min}$ rest, $2 \mathrm{~min}$ jogging $(5 \mathrm{~m} / \mathrm{h}), 1 \mathrm{~min}$ rest, $2 \mathrm{~min}$ running $(7 \mathrm{~m} / \mathrm{h}), 1$ min rest, 1 min arbitrary movement. The ECG-based reference HR was recorded in order to assess the performance of the second phase of the TifMA algorithm.

All PPG data were preprocessed by a sixth-order infinite impulse response (IIR) band pass filter with cutoff frequencies of 0.1 and $10 \mathrm{~Hz}$. Zero-phase forward and reverse filtering was applied to account for the nonlinear phase of the IIR filter. 
TABLE IV

Performance Metrics (Mean \pm Std.) of Our Proposed TifMA mNA Detection Algorithm Versus Other Methods

\begin{tabular}{|c|c|c|c|c|c|}
\hline & & TifMA & Hjorth & TDV & KSE \\
\hline \multirow[t]{3}{*}{ Lab. head } & Accuracy & $95.7 \pm 0.82$ & $72.5 \pm 10.74^{*}$ & $93.4 \pm 3.50^{*}$ & $83.1 \pm 7.31^{*}$ \\
\hline & Sensitivity & $93.0 \pm 5.75$ & $47.2 \pm 28.80^{*}$ & $88.8 \pm 7.90^{*}$ & $56.6 \pm 17.88^{*}$ \\
\hline & Specificity & $96.6 \pm 1.48$ & $84.4 \pm 4.63^{*}$ & $96.7 \pm 300$ & $91.5 \pm 1.14^{*}$ \\
\hline \multirow[t]{3}{*}{ Umass. head } & Accuracy & $95.3 \pm 1.34$ & $69.5 \pm 21.83^{*}$ & $89.9 \pm 1.45^{*}$ & $85.1 \pm 8.34^{*}$ \\
\hline & Sensitivity & $90.8 \pm 2.83$ & $53.8 \pm 26.05^{*}$ & $84.6 \pm 2.89^{*}$ & $68.7 \pm 17.72^{*}$ \\
\hline & Specificity & $98.7 \pm 1.07$ & $84.5 \pm 8.83^{*}$ & $94.3 \pm 3.95^{*}$ & $86.4 \pm 7.82^{*}$ \\
\hline \multirow[t]{3}{*}{ Lab. finger } & Accuracy & $97.5 \pm 1.50$ & $91.1 \pm 6.87^{*}$ & $94.4 \pm 3.30^{*}$ & $58.5 \pm 20.67^{*}$ \\
\hline & Sensitivity & $96.4 \pm 2.34$ & $83.5 \pm 2104^{*}$ & $94.7 \pm 3.40$ & $34.6 \pm 12.23^{*}$ \\
\hline & Specificity & $98.1 \pm 1.43$ & $96.2 \pm 3.02$ & $94.7 \pm 3.00^{*}$ & $86.3 \pm 15.65^{*}$ \\
\hline \multirow[t]{3}{*}{ Umass. finger } & Accuracy & $94.3 \pm 1.64$ & $71.0 \pm 18.96^{*}$ & $89.6 \pm 2.34^{*}$ & $88.3 \pm 2.68^{*}$ \\
\hline & Sensitivity & $88.5 \pm 2.23$ & $41.1 \pm 27.57^{*}$ & $85.2 \pm 3.75^{*}$ & $71.5 \pm 8.83^{*}$ \\
\hline & Specificity & $96.9 \pm 1.86$ & $88.4 \pm 7.52^{*}$ & $95.1 \pm 0.79$ & $93.6 \pm 1.52^{*}$ \\
\hline
\end{tabular}

$\left({ }^{*}\right)$ indicates statistically significant difference $(p<0.05)$ between TifMA and the others.

\section{B. Reference Signal for MNA Detection}

Many recent publications on MNA detection used human visual inspection by experts who were familiar with PPG, and their decisions are regarded as the gold standard for marking MNA-corrupted data [22], [23], [27]. In our work, we also use human visual inspection to establish a MNA reference for our datasets. Three inspectors individually marked MNA-corrupted portions of the PPG. Disagreements about the marked portions were resolved by majority votes. Cohen's $\kappa$ was used to determine if there was agreement between three inspectors' judgments on whether PPG segments were declared to be clean or corrupted. For each dataset, the average Cohan's $\kappa$ was reported in Table II over three runs computed from each distinct inspector pair. Overall, the Cohan's $\kappa$ showed substantial agreement between the inspectors with $95 \%$ CI and $p<0.0005$.

\section{Reference Signal for Data Usability Detection}

A usability index (UI) was calculated to measure the usability of MNA-corrupted segments of PPG recordings. In order to verify the performance of the index, it was compared to the reference usability measurements. The reference was determined according to the deviation of reference HR from the chest ECG, and the estimated HR obtained from phase 2 of the TifMA algorithm.

\section{TifMA}

As mentioned above, the TifMA algorithm consists of two major phases: 1) MNA detection; and 2) usability detection. Both phases of the algorithm were developed based on a TF technique named VFCDM. VFCDM is a method for estimating the TFS of a time-varying signal. This method was shown to provide concomitant high time and frequency resolution as well as preservation of the amplitude distribution of the signal [31]. VFCDM has two steps: 1) constructing an initial TFS (iTFS) using a method developed in our laboratory, termed fixed frequency complex demodulation (FFCDM); and 2) doing further complex demodulation (CDM) using the center frequencies of the iTFS to obtain even more accurate TFS and amplitude of the

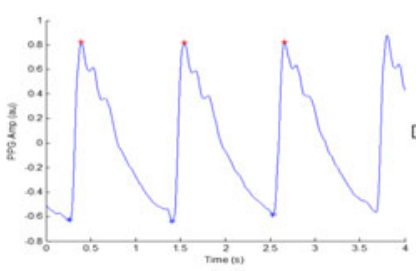

(a)

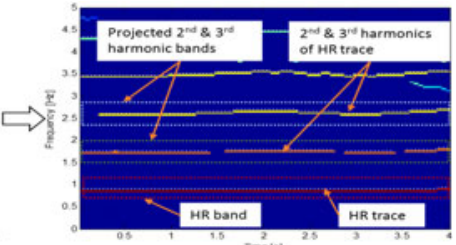

(b)

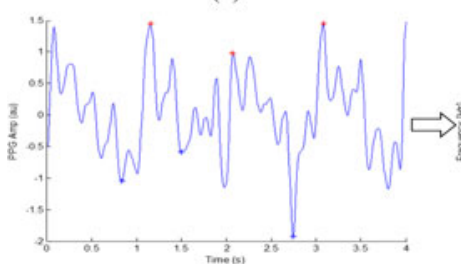

(c)

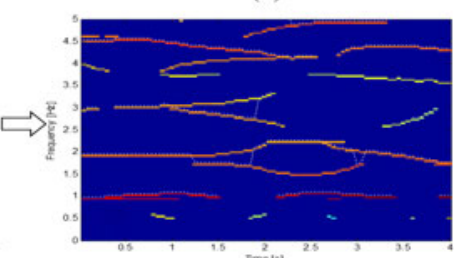

(d)
Fig. 1. TFS produced by VFCDM on a $4 \mathrm{~s}$ PPG window. The white rectangular boxes point to the fundamental band and its second and third projected harmonic bands. (a) Clean PPG. (b) TFS of clean PPG. (c) Corrupted PPG. (d) TFS of corrupted PPG.

TFS. Succinct details of VFCDM are provided in the Appendix, but for further details, the reader is referred to [31].

\section{Methodology}

\section{A. TifMA Phase 1: MNA Detection}

In the first phase of the algorithm, the TFS of PPG is used to extract features that contribute to discriminating between MNA-corrupted PPG data and clean data segments. Fig. 1(a) and (b) illustrates an example of VFCDM-TFS applied to $4 \mathrm{~s}$ segments of clean and MNA-corrupted PPG data. It can be observed from this figure that the TFS of a corrupted PPG segment has different characteristics compared to the TFS of a clean segment. The HR trace and its harmonics are distinguishable in the clean TFS while MNAs distort the HR and its harmonics' traces in the TFS of MNA-corrupted PPG data. In this paper, $\mathrm{FM}_{1}, \mathrm{FM}_{2}$, and $\mathrm{FM}_{3}$ refer to the HR trace and two of its harmonic traces, while to refer to the corresponding spectral power, the terms $\mathrm{AM}_{1}, \mathrm{AM}_{2}$, and $\mathrm{AM}_{3}$ are used. $\mathrm{FM}$ and $\mathrm{AM}$ stand for frequency modulation and amplitude modulation, respectively. 
Since respiratory-induced fluctuations in PPG are highly dynamic and not trivial to characterize in a TFS, regions associated with respiratory frequencies are removed from the TFS by setting their powers to zero. The respiratory frequencies are defined to be between 0 and $0.5 \mathrm{~Hz}$. Our algorithm first determines the dominant frequency in the PPG segment, termed $f_{1}$, predicated upon the assumption that PPG is dominantly driven by cardiac cycles. To determine instantaneous $f_{1}$, total power within the narrow-band spectral window $W_{k}=\left[f_{k}-\mathrm{BW}, f_{k}+\mathrm{BW}\right]$ is computed, where $\mathrm{BW}=0.2 \mathrm{~Hz}$ is the bandwidth of the band and $f_{k}$ is the center frequency of the window ranging from 0.6 to $2.4 \mathrm{~Hz}$ with increment of $0.1 \mathrm{~Hz}$. BW was determined based on an assumption that within a short time HR does not fluctuate more than 12 beat per minute. $f_{1}$ is estimated as $f_{k}$ at which $W_{k}$ is maximum.

The TFS of the segment is normalized by the total power in the $f_{1}$ band. From the normalized TFS, we then extract $\mathrm{FM}_{1}$ and $\mathrm{AM}_{1}$ within $\left[f_{1}-\mathrm{BW}, f_{1}+\mathrm{BW}\right]$. Note that each point in the TFS has three instantaneous values: time, frequency, and spectral power. The maximal power in each time instance is taken to form $\mathrm{AM}_{1}=\mathrm{AM}_{1}(t): t=1, \ldots, N-2$ where $N$ is the number of data points in the PPG segment. Its corresponding $\mathrm{FM}_{1}$ is also extracted. Once located, $\mathrm{AM}_{1}$ is removed from the TFS by setting its power to zero. Similarly, $\left\{\mathrm{AM}_{2}, \mathrm{FM}_{2}\right\} \in\left[2 f_{1}-\mathrm{BW}, 2 f_{1}+\mathrm{BW}\right]$ and $\left\{\mathrm{AM}_{3}, \mathrm{FM}_{3}\right\} \in\left[3 f_{1}-\mathrm{BW}, 3 f_{1}+\mathrm{BW}\right]$ are found and removed. Note that the algorithm assumes that corrupted PPG segments would exhibit irregularity in the time series waveform. Thus, the TFS of a corrupted segment would have broadband dynamics. In this case, the proposed fundamental frequency estimation method would probably yield inaccurate result. However, an arbitrary estimation of HR (fundamental frequency and its harmonics) in such cases would remove only a portion of the noise power within the defined bands, but would retain the rest.

From the extracted TFS and $\left\{\mathrm{FM}_{i}, \mathrm{AM}_{i}: i=1,2,3\right\}$, three TF features were derived to quantify the noise level between clean versus corrupted PPG segments. These will be henceforth termed, collectively, the TF features.

1) Residual Noise Power ( $\left.P_{\text {noise }}\right)$ : After extracting the first three dominant traces, the remaining power in the TFS is considered the residual noise power $P_{\text {noise }}$ and is denoted as follows:

$$
P_{\text {noise }}=P_{\mathrm{TFS}}-\sum_{i=1}^{3} \sum_{t} \mathrm{AM}_{i, t}
$$

where $P_{\mathrm{TFS}}$ is the total power in the TFS. In a clean PPG segment as illustrated in Fig. 1(b), the first three harmonics would be located within the predetermined narrow band. Thus, extracting their power would effectively remove most of the spectral power from the TFS. The remaining noise power would be negligibly small. On the other hand, artifacts in the corrupted PPG segment produce spectral power at various frequency locations which are often not associated with the harmonics' frequency bands as illustrated in Fig. 1(d). Some of this spectral power is outside of the bands and/or there are multiple power within a band. Therefore, the power would not be extracted which in turn yields high $P_{\text {noise }}$ level.

2) Projected Frequency Modulation Difference ( $\left.d f_{\mathrm{FM}}\right)$ : Projected difference is defined as the difference in frequency between the fundamental HR trace and its harmonic traces and is computed as follows:

$$
d f_{\mathrm{FM}}=\sum_{i=2}^{3} \sum_{t}\left|\mathrm{FM}_{\mathrm{i}, t}-i \times \mathrm{FM}_{1, t}\right| .
$$

Similar to the previous assumption, the frequency locations of the harmonic traces are expected to be proportional to that of the fundamental trace, which would result in a low $d f_{\mathrm{FM}}$ for a clean segment. For an artifact-corrupted segment, the proportionality in the frequency of the harmonics would no longer hold, thus driving $d f_{\mathrm{FM}}$ value to be high.

3) HR Frequency Difference $\left(d f_{\mathrm{HR}}\right)$ : HR frequency difference is defined as the difference between the fundamental frequency modulation $\mathrm{FM}_{1}$ and $\mathrm{HR}$ computed from the timedomain peak calculation. This feature measures the agreement between the fundamental frequencies detected from the TFS and from the time-domain signal. It is assumed that the frequencies would be in agreement in a clean PPG segment. In a noise-corrupted segment, however, there could even be large differences in the values. We compute $d f_{\mathrm{HR}}$ as follows:

$$
d f_{\mathrm{HR}}=\sum_{t}\left|\mathrm{FM}_{1, \mathrm{t}}-\operatorname{median}\left(\frac{1}{P P}\right)\right|
$$

where PP (second) are the peak-to-peak intervals in a PPG segment.

As mentioned above, the results of the MNA detection phase of TifMA were compared to three other existing MNA detection algorithms: the Hjorth [26], kurtosis-SE [23] and TDV-SVM approach, an approach recently developed in our laboratory [27].

Feature extraction from PPG data was done on a sliding window segment of length $L_{1}=8 \mathrm{~s}$ with $50 \%$ overlap. Each segment was transformed using VFCDM into TFS, of which only the middle portion of length $L_{2}=4 \mathrm{~s}$ was considered for further processing. This is because, as shown in Table III, the middle $4 \mathrm{~s}$ of data out of the full $L_{1}=8 \mathrm{~s}$ provided the best accuracy in detection of MNAs.

To accurately pinpoint the time occurrence of MNAs, we implemented a trace-back strategy, which is triggered when the

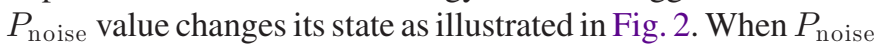
goes from lower than a threshold value of 0.15 to greater than 0.15 , the trace-back algorithm updates the TF features three times by shifting backward a second at each time instant. For example, in Fig. 2(a), $P_{\text {noise }}$ changes to a value that is greater than 0.15 for the time duration $4-8 \mathrm{~s}$. The trace-back scheme would call the VFCDM routine to compute new TF features for the back-shifted segments at time durations starting at 3-7, 2-6 s, and ending at 1-5 s. As detailed above, our VFCDM algorithm is designed to indicate that a segment is corrupted even if only $1 \mathrm{~s}$ of the $4 \mathrm{~s}$ duration contains MNAs. Hence, since the 3-7 s segment is determined to be corrupted, it allows us to deduce that data at the $8 \mathrm{~s}$ time point are also corrupted. The same logic applies to the 2-6 and 1-5 s segments. An example 


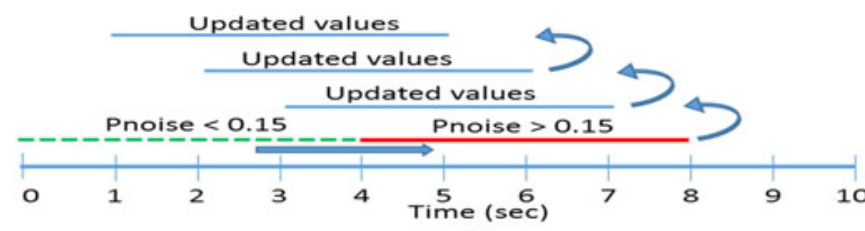

(a)

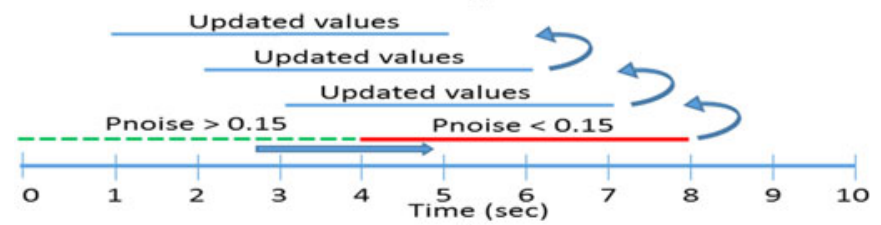

(b)

Fig. 2. Trace-back strategy to find (a) start and (b) end points of MNA.
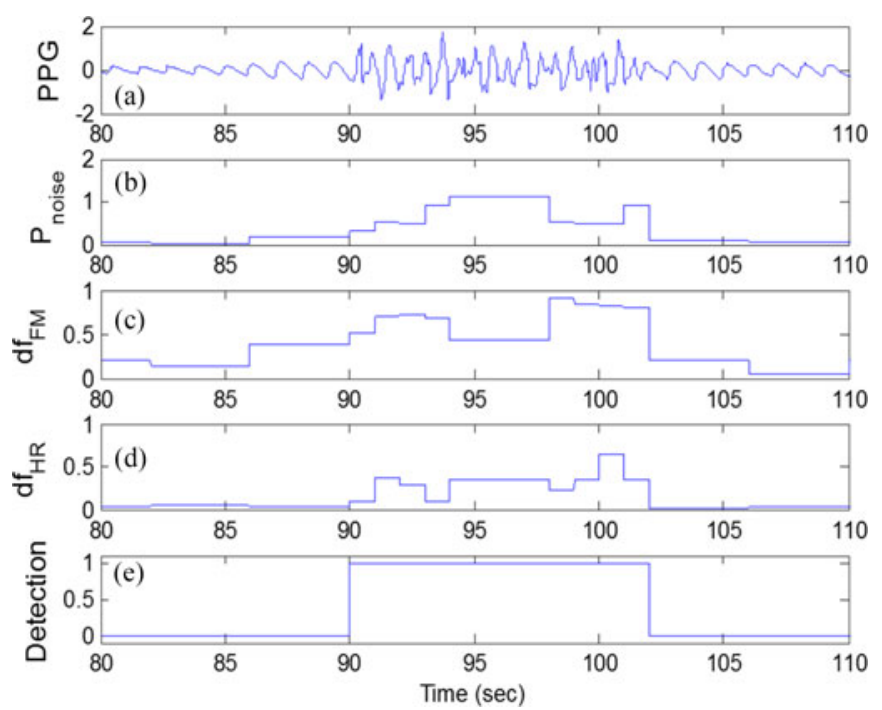

Fig. 3. Example of MNA detection using TifMA. (a) PPG signal corrupted with MNA. (b)-(d) TF features from TifMA. (e) Detection decision of TifMA.

of the track-back strategy is illustrated in Fig. 2 where: (a) shows TF features are updated at a possible starting point of an MNAcorrupted segment; and (b) shows TF features are updated at a possible ending point of an MNA-corrupted segment.

The SVM was applied to build a decision boundary to detect MNA-corrupted PPG segments from clean data. The SVM is widely used for classification and regression analysis due to its accuracy and robustness to noise [12], [32].

Fig. 3 shows a representative illustration of the performance of our proposed MNA detection approach. Fig. 3(a) displays a preprocessed PPG signal containing both clean and MNAcorrupted periods. Fig. 3(b)-(d) depicts the corresponding TF features described above. As shown, the TF features have low values for the clean portion of the data whereas they are high when MNAs occur. The TF features are used for the SVM classifier to determine whether the given segment is clean or corrupted. Table IV shows the classification results.

A representative example of the MNA detection comparing all of the aforementioned methods is illustrated in Fig. 4.

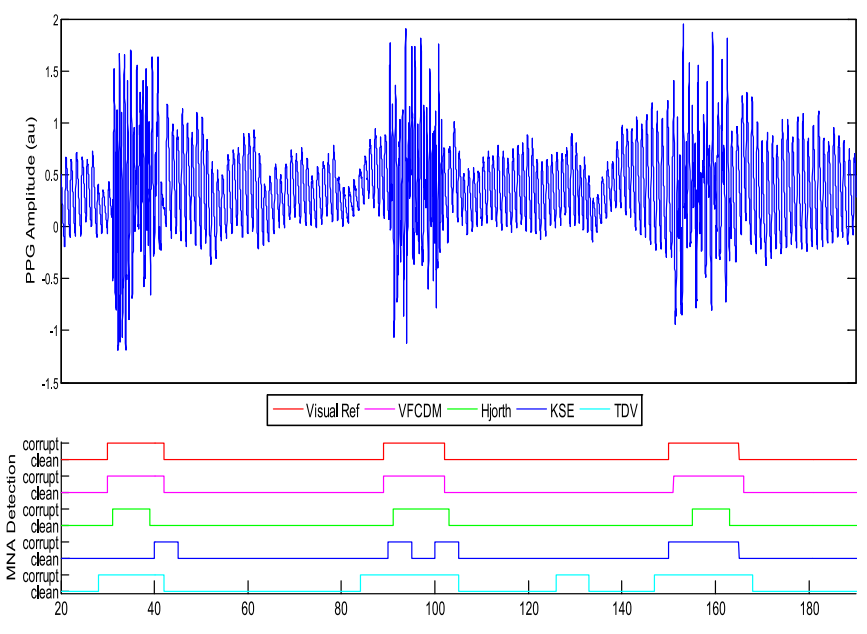

Fig. 4. Example of MNA detection using our VFCDM method versus other methods: Hjorth, time-domain, and kurtosis-SE. The pulse-like traces are the MNA reference and detection results from the feature sets. High value indicates detected MNA, otherwise clean PPG signal.

\section{B. TifMA Phase 2: Usability Detection}

In Fig. 1, we show that when the PPG signal is corrupt, the HR trace and its harmonics in TFS also exhibit distortion, but the amount of distortion is not uniform. For example, the severity of MNA has a direct correspondence to the accuracy of the HR estimates. Thus, a proper way to determine if a corrupted signal is usable or not for accurate HR estimation is to extract the tracing of HR values from TFS and analyze the HR time series for missing values and the presence of abrupt changes in HR estimation, as these events often result in unusable data. In order to examine this idea, a dataset with slow and rapidly changing HR scenarios was analyzed. The data were recorded from subjects while walking, jogging, and running on a treadmill. Fig. 5 illustrates a $7.5 \mathrm{~min}$ segment $(0.5 \mathrm{~min}$ rest, $2 \mathrm{~min}$ walking $(3 \mathrm{~m} / \mathrm{h}), 1 \mathrm{~min}$ rest, $2 \mathrm{~min}$ jogging $(5 \mathrm{~m} / \mathrm{h}), 1 \mathrm{~min}$ rest, $1 \mathrm{~min}$ running $(7 \mathrm{~m} / \mathrm{h})$ ) from the 9 min recording of a representative subject. Fig. 5(a) and (b) shows the recordings from a Holter monitor and concomitant PPG recording, respectively. The reference HR extracted from ECG data is shown in Fig. 5(c). It can be observed from the reference HR [see Fig. 5(c)] that HR varies from low to high values ranging from 80 to $160 \mathrm{bpm}$. Fig. 5(d) shows MNA detection results using the first phase of our algorithm. Fig. 6 represents the VFCDM-TFS of the corresponding PPG data in Fig. 5(b). One can identify the tracing of HR in TFS which begins to show distortion during the time stamps of 260-340 s and 440-500 s. By applying the MNA detection procedure of TifMA, we can observe that motion-related data are truly marked as MNA-corrupted segments. The next step is to investigate if any portion of these MNA-corrupted segments is usable for reliable HR estimation.

The following describes the second phase of the TifMA algorithm, which is discrimination between nonusable and usable portions of the data segments identified as corrupted. The usability detection stage is comprised of three steps: 1) TFS filtering; 2) HR tracking and extraction; and 3) UI measurement. Fig. 7 


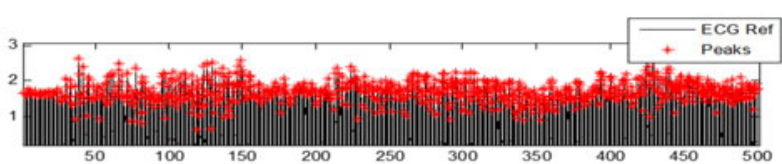

(a)

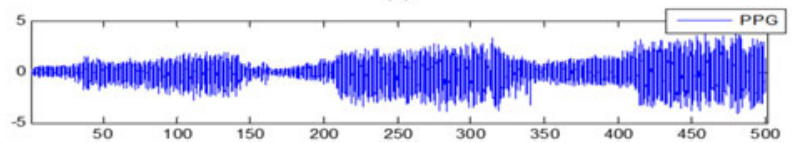

(b)

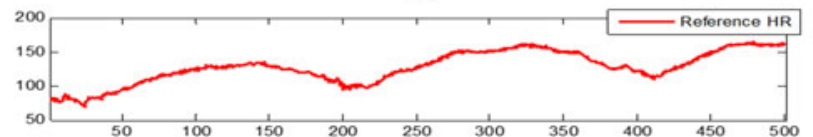

(c)

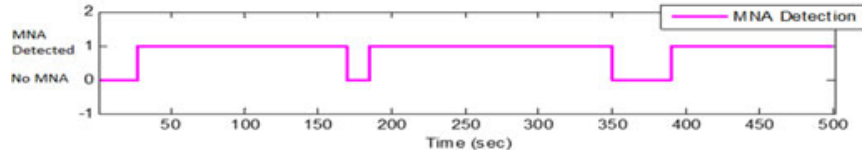

(d)

Fig. 5. Subject \#7 from dataset (3): (a) reference ECG, (b) PPG recording, (c) estimated reference HR from reference ECG, and (d) TifMA detection ("0": no MNA, "1": MNA detected).

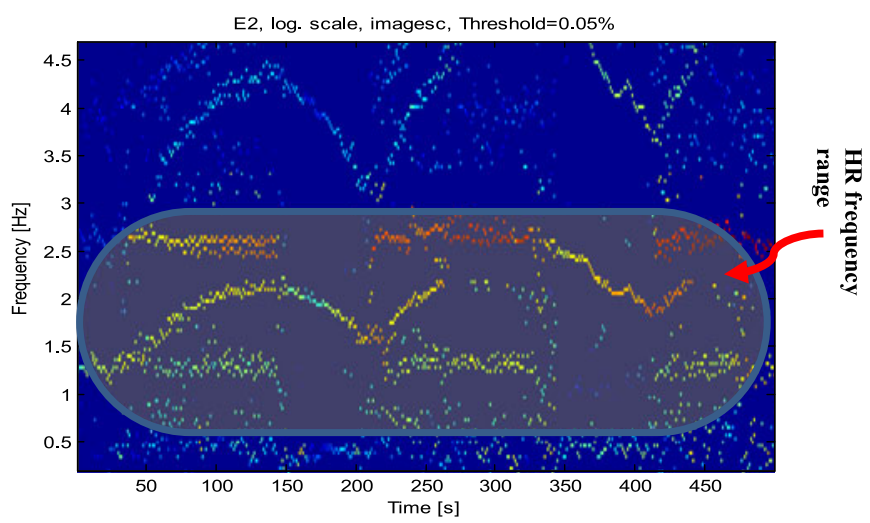

Fig. 6. VFCDM Time-Frequency Spectrum of Subject \#7 from dataset (3).

represents spectral plots of three different scenarios that occurred in Fig. 6. In Fig. 7(a) and (b), the true HR frequency is close to the first and second highest peak, respectively, while in Fig. 7(c) the true HR frequency is far removed from the dominant peak in the spectrum.

We can assume that as long as a segment of PPG data is clean, the true HR frequency belongs to the frequency component with the highest power (peak) in each column of the TFS matrix. On the other hand, when the data are corrupted by MNA, the true HR frequency may shift to the next-highest peak in the spectrum [e.g., Fig. 7(b)]; for severe MNA, the scenario of Fig. 7(c) may occur. Hence, we proposed to design a TFS filtering step to look for the first two highest spectral peaks and the corresponding frequencies in TFS. Hence, the original TFS (see Fig. 6) can be filtered to keep only the two largest spectral peaks.

The next step is to extract continuous HR. The HR tracking procedure is straightforward, as follows. Assuming that the initial HR is devoid of MNA, the HR in each $8 \mathrm{~s}$ window is extracted by comparing the two largest peaks to the previous HR

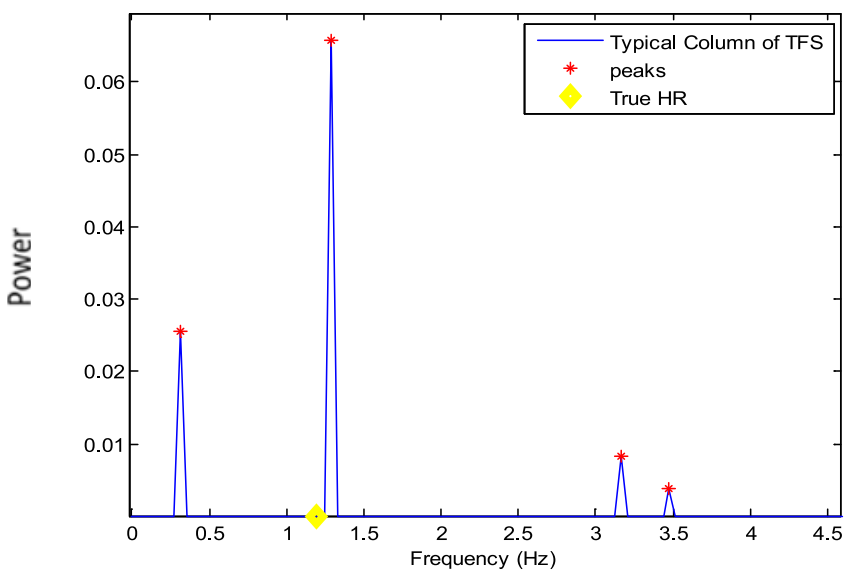

(a)

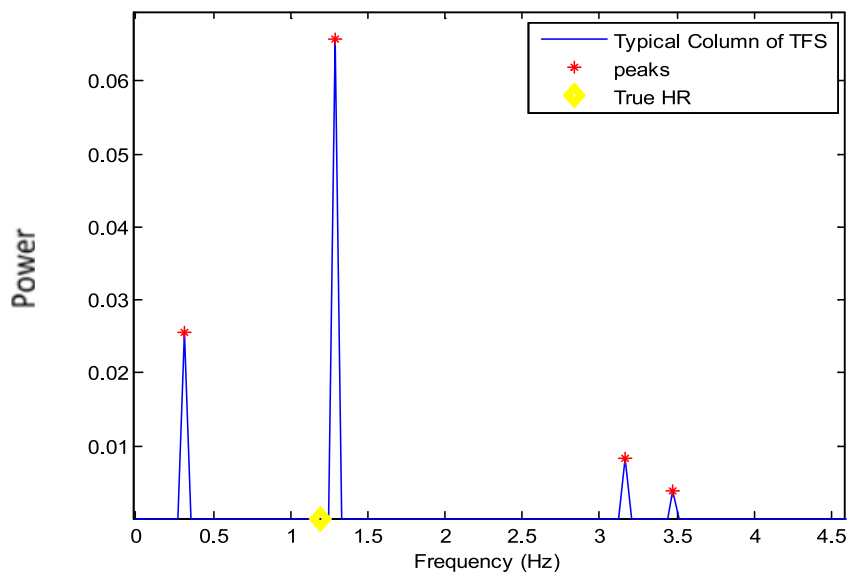

(b)

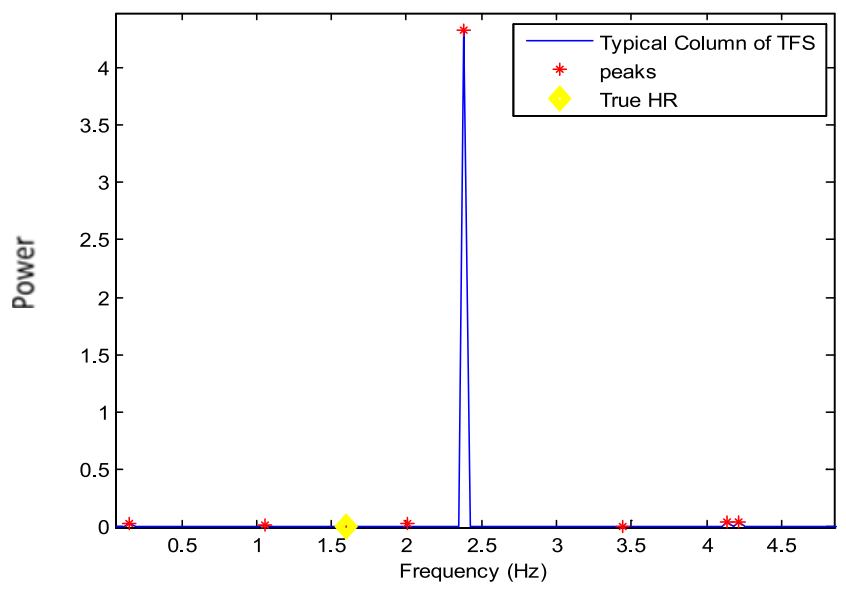

(c)

Fig. 7. Examples of usable and nonusable PPG data. (a) Usable: true $\mathrm{HR}$ frequency (yellow circle) is close to the highest peak of spectrum. (b) Usable: true HR frequency (yellow circle) is close to the second-highest peak. (c) Nonusable: true HR (yellow circle) is not close to any prominent peaks in the spectrum.

value. If either of the peaks are within $0.08 \mathrm{~Hz}(5 \mathrm{bpm})$ from the most recent value of HR, it is chosen as usable; if the value deviates more than $0.17 \mathrm{~Hz}(10 \mathrm{bpm})$, it is considered nonusable. Fig. 8(a) represents the estimated $8 \mathrm{~s}$ moving averaged HR from TFS compared to the reference HR from ECG. Calcu- 


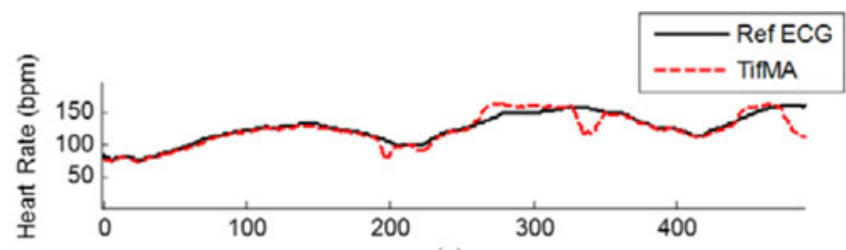

(a)

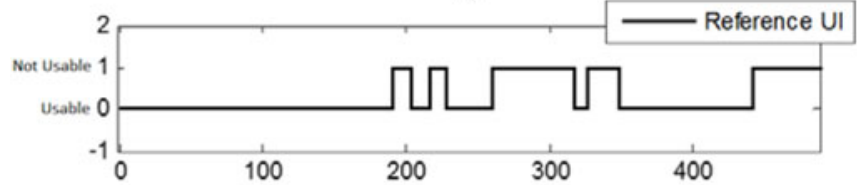

(b)

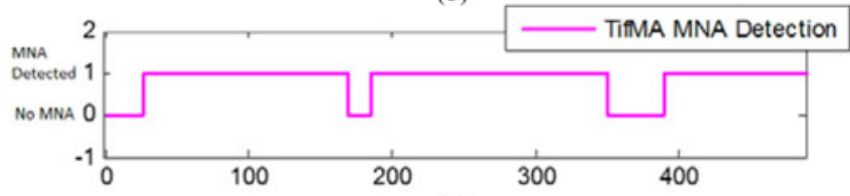

(c)

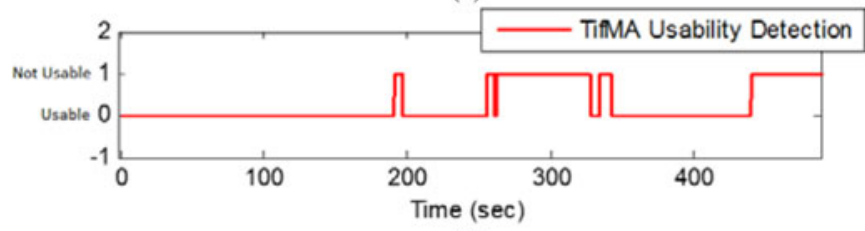

(d)

Fig. 8. Reference UI. (a) Comparison of TifMA-estimated HR to reference HR from ECG. (b) Reference UI as an indicator of the fidelity of HR estimate from TFS during motion. (c) TifMA MNA detection result. (d) TifMA usability detection, " 0 " indicates no MNA and "1" indicates MNA detected.

lation of the reference UI (useable if within $5 \mathrm{bpm}$, otherwise not usable) is performed according to the deviation of TifMAestimated HR from the true reference HR [see Fig. 8(a) and (b)]. Fig. 8(c) shows the results of MNA detection, the first phase of the algorithm. It can be observed from this figure that segments of PPG recordings during movement (walking/jogging/running) have been mostly detected as being corrupted by MNA. Fig. 8(d) shows the UI derived by the second phase of TifMA. One can observe by comparing the reference UI [see Fig. 8(b)] and TifMA's UI [see Fig. 8(d)] that TifMA detects the usable/nonusable portions of the data for HR estimations quite well. More importantly, we observe that significant portions of the corrupted data [see Fig. 8(c)], as identified according to the first phase of our algorithm, are found to be usable as shown in Fig. 8(d). The accuracy, specificity, and sensitivity of the proposed algorithm on all ten of the recordings from dataset \#5 are presented in the following section.

The overall TifMA algorithm flowchart is depicted in Fig. 9.

\section{Results}

We evaluated the performance of the TifMA algorithm on five datasets. The first four datasets (laboratory controlled, and hospital patients with motion-corrupted PPGs) were used to measure the performance of the first phase of the algorithm (MNA detection) and the fifth (treadmill experiment) was used to evaluate the second phase of the algorithm (usability detection).

\section{A. MNA Detection Results}

Leave-one-out cross validation was adopted to evaluate the performance of the MNA detection phase [33].

In addition to accurate MNA detection, the other attractive feature of our proposed algorithm is that it is able to accurately locate the start and end points of MNA occurrences. Accurate detection of the start and end times of the MNA-corrupted segments is important for the subsequent reconstruction of the MNA-corrupted data as we do not want to miss the MNA portion of data for reconstruction or avoid having to reconstruct the noisy portion of the PPG when the segment is designated to be clean.

To evaluate the algorithm's effectiveness in pinpointing the start and end times of the MNAs, we computed the time difference of start and end points between the visual reference and detection algorithm's results. The time difference is termed detection transition time, DTT, which reflects how accurately on average an MNA algorithm detects the start and end times of the MNAs. Table $\mathrm{V}$ provides a comparison DTT values from the TifMA algorithm and from other detection algorithms. As shown in Table V, our algorithm's detection accuracy of the duration of the MNAs is significantly better than the accuracy of the three other methods we compared. Our algorithm's DTT is less than $1 \mathrm{~s}$ off whereas the second best algorithm, the Hjorth, is off by more than $2 \mathrm{~s}$ and the least accurate method, the KSE, is off by more than $4 \mathrm{~s}$.

\section{B. Usability Detection Results}

By applying the first phase of the TifMA algorithm, we showed that the algorithm is able to determine if a PPG data segment is clean or is corrupted with MNA. However, being corrupted by movement does not necessarily suggest that the entire noisy data segment is nonusable in terms of HR estimation. It was shown that HR frequency changes can be tracked using VFCDM TF plot as long as the signal is usable and the level of motion corruption is not too severe.

Nonusable data can usually be attributed to abrupt or fast movements that make tracking of frequency especially difficult. To this end, the second phase of the algorithm was tested on PPG recordings from ten subjects who performed a 9 min experiment, during which they walked, jogged, and ran. The data initially were fed into the MNA detection algorithm and when a corrupted data segment was obtained, the second phase of the algorithm performed usability determination of data, that is whether the corrupted signal is usable or not for HR estimation. Table VI presents the accuracy, sensitivity, and specificity of the usability detection calculated by comparing to the reference UI.

As can be seen from Table VII, TifMA initially identified $48 \%$ of the data corrupted by MNA as nonusable HR data, but after the second phase of our algorithm, only $10 \%$ of PPG recordings during the treadmill experiment were determined to be so severely corrupted that they could not be used for reliable HR estimation. 


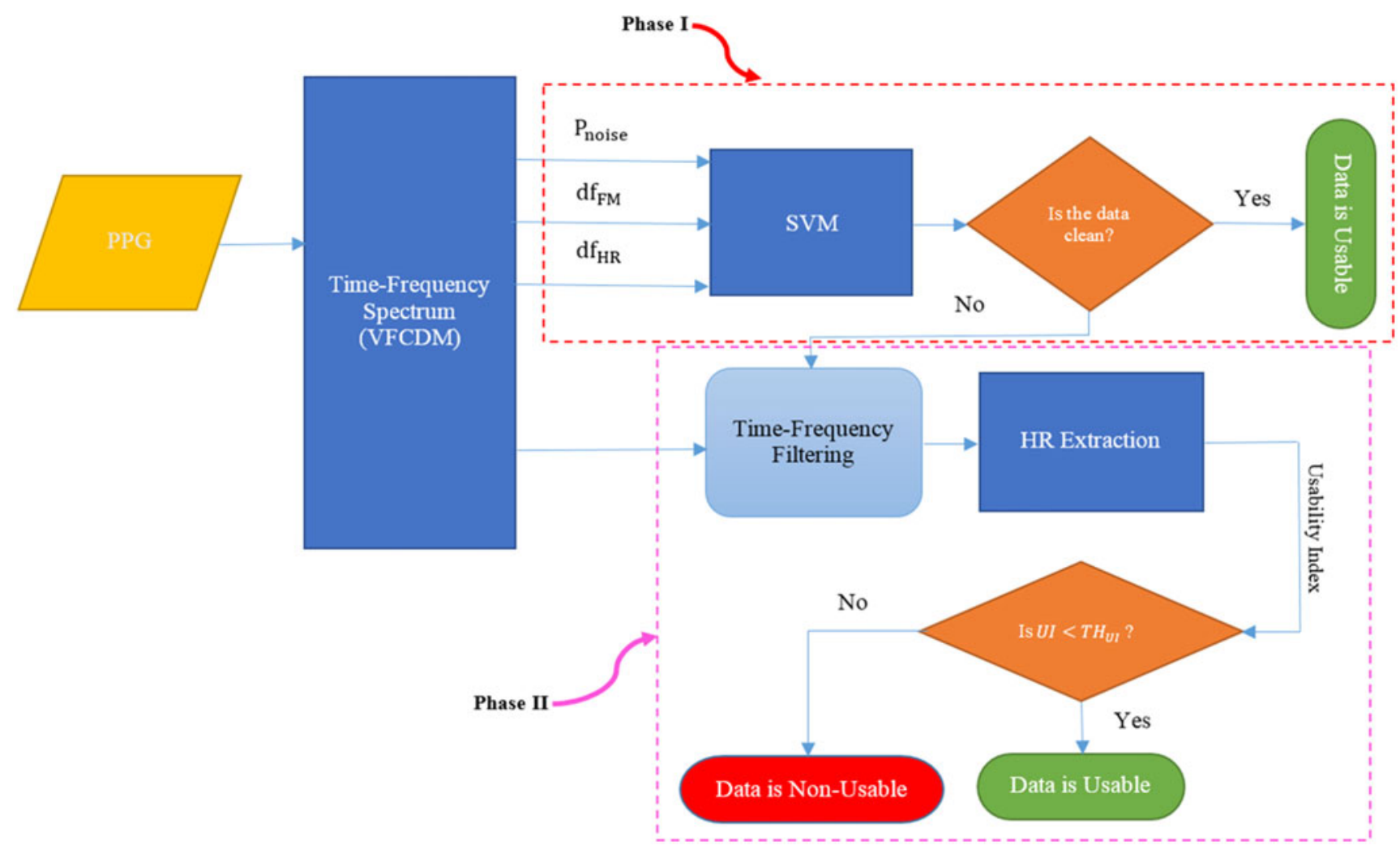

Fig. 9. TifMA algorithm flowchart.

\section{DISCUSSION}

We propose a novel method to overcome MNAs in PPG data, that we term "TifMA," that makes use of the dynamic characteristics of the signal. The algorithm is implemented in two phases: 1) MNA detection; and 2) usability detection. The efficacy of the detection phase was validated using contrived motion data from healthy subjects and unconstrained MNA data from participants recruited in a hospital setting. The second phase of the algorithm was tested on HR-varying scenarios during which subjects were asked to walk, jog, and finally run for a total of $9 \mathrm{~min}$ on a treadmill. To achieve MNA detection, the method takes advantage of several key features associated with MNAs derived from the VFCDM-based TFS. By transforming the PPG time series into the TF domain, we were able to better capture time-varying characteristics of the MNAs. Specifically, we recognized that clean PPG signal dynamics are largely concentrated at the HR and its harmonic frequency bands. Hence, we surmised that the presence of large amplitudes in other frequency bands must be associated with MNAs.

The detection accuracy on both laboratory-controlled and clinical datasets using TifMA outperformed the other three detection methods: Hjorth, TDV-SVM, and KSE. We compared each method's performance based on their own unique feature selection by evaluating the area under the receiver operating characteristic curve. The areas under the curves (AUCs) showed that our TF features provided the highest values, with AUCs > 0.89 for both finger data and forehead-recorded PPG. Concomitantly, the accuracy, sensitivity, and specificity values of our
TABLE V

MEAN \pm STD. DTT OF TIFMA AND OTHER METHODS

\begin{tabular}{lc}
\hline \hline Algorithm & DTT (second) \\
\hline TifMA & $0.91 \pm 0.59$ \\
Hjorth & $2.17 \pm 0.37$ \\
KSE & $4.24 \pm 2.42$ \\
TDV & $2.75 \pm 0.96$ \\
\hline
\end{tabular}

TABLE VI

TifMA Usability Detection Performance

\begin{tabular}{|c|c|c|c|c|}
\hline Subjects & Dataset (5) & Accuracy & Sensitivity & Specificity \\
\hline 1 & & 0.89 & 0.94 & 0.92 \\
\hline 2 & & 0.93 & 0.90 & 0.88 \\
\hline 3 & & 0.95 & 0.92 & 0.90 \\
\hline 4 & & 0.93 & 0.91 & 0.96 \\
\hline 5 & & 0.91 & 0.93 & 0.84 \\
\hline 6 & & 0.91 & 0.90 & 0.90 \\
\hline 7 & & 0.93 & 0.92 & 0.91 \\
\hline 8 & & 0.95 & 0.94 & 0.92 \\
\hline 9 & & 0.94 & 0.96 & 0.94 \\
\hline 10 & & 0.90 & 0.97 & 0.90 \\
\hline mean \pm std & & $0.92 \pm 0.02$ & $0.93 \pm 0.02$ & $0.90 \pm 0.03$ \\
\hline
\end{tabular}

proposed method were significantly higher than those of the other methods, as indicated in Table V.

Most time-domain motion noise artifact detection techniques [20], [21], [27] (e.g., accelerometer-based MNA detection) are not always accurate when they are used for HR or respiratory rate monitoring applications. Fig. 10 illustrates this point. Specifically, Fig. 10(a) illustrates an example of MNA analysis 
TABLE VII

(Continued) TifMA Usability Detection Performance

\begin{tabular}{|c|c|c|c|}
\hline Subjects & Dataset (5) & $\begin{array}{l}\text { Non-usable \% (After TifMA } \\
\text { phase1 before phase 2) }\end{array}$ & $\begin{array}{c}\text { Non-usable \% } \\
\text { (After TifMA } \\
\text { Phase 2) }\end{array}$ \\
\hline 1 & & $63.06 \%$ & $20.00 \%$ \\
\hline 2 & & $43.27 \%$ & $14.56 \%$ \\
\hline 3 & & $38.13 \%$ & $17.56 \%$ \\
\hline 4 & & $21.85 \%$ & $1.33 \%$ \\
\hline 5 & & $48.19 \%$ & $3.89 \%$ \\
\hline 6 & & $60.38 \%$ & $5.33 \%$ \\
\hline 7 & & $74.21 \%$ & $24.78 \%$ \\
\hline 8 & & $41.39 \%$ & $3.67 \%$ \\
\hline 9 & & $44.73 \%$ & $6.89 \%$ \\
\hline 10 & & $40.66 \%$ & $11.67 \%$ \\
\hline mean \pm std & & $47.58 \% \pm 14.82 \%$ & $10.97 \% \pm 7.98 \%$ \\
\hline
\end{tabular}
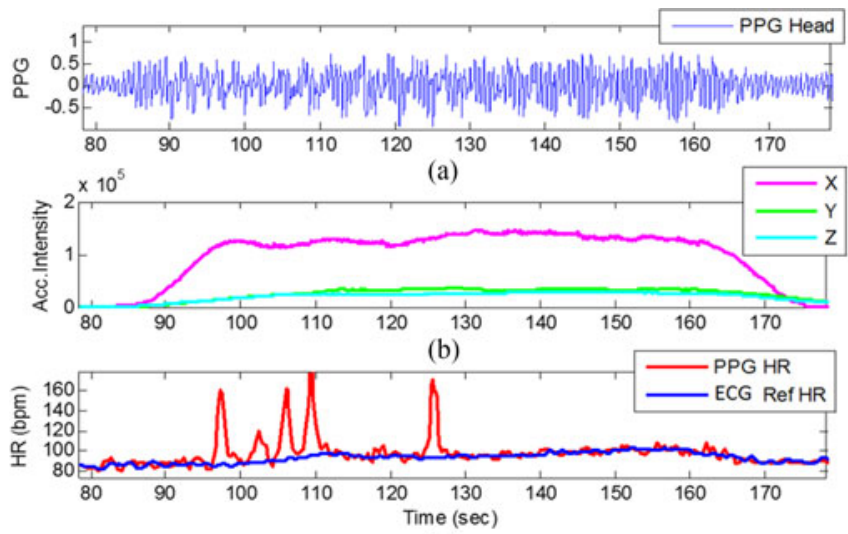

(c)

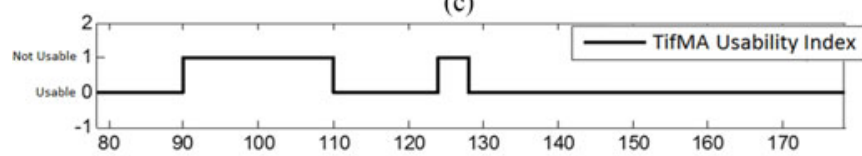

(d)

Fig. 10. Example showing accelerometer versus TifMA-based methods' performance on a typical PPG signal recorded from a subject during walking.

of PPG recordings from a walking subject. Fig. 10(b) represents the accelerometer intensity of triaxial accelerometer recordings. Movement intensity from three axial accelerometer signals is calculated by taking the moving average of the squared derivative of accelerometer raw data [21]. Fig. 10(c) shows the HR estimations from the PPG data, and reference HR estimated from the clean reference ECG. It can be observed in Fig. 10(b) that the accelerometer is very sensitive to movement; as soon as the subject starts to walk (at $\sim 90$ s), it shows an abrupt increase in the accelerometer signal intensity. However, in Fig. 10(c), the HR from PPG can be accurately estimated in some section of movement. By applying the TifMA algorithm on this segment of PPG data, the algorithm is able to discriminate between the usable and nonusable segments of PPG for HR estimations [see Fig. 10(d)].

It can be seen from the aforementioned example that if accelerometers' intensity values are solely used for MNA detection, the entire data segment from 90 to $170 \mathrm{~s}$ is deemed to be not useable. Certainly, the time windows at $95-115 \mathrm{~s}$ and at 125
127 s can be considered not usable since the PPG-estimated HR significantly deviates from the reference ECG HR. However, for a time segment from 130 to $160 \mathrm{~s}$, the HR estimation from PPG is very close to that of the reference ECG even though the accelerometers' intensity is as high as it is in the time window between 95 and $115 \mathrm{~s}$.

Therefore, just relying on accelerometers can lead to incorrect nonusable data determination in the time segment between 130 and 160 s, as shown in Fig. 10(b) and (c). Note, however, with our proposed TifMA algorithm, it can accurately determine the start and end points of usable and nonusable segments of data, as shown in Fig. 10(d). Note that other detection methods including those compared (e.g., Table V) in this work do not have data usability capability. Hence, we are not able to provide comparison of their performances against our algorithm.

The eventual aim of our proposed algorithm is to detect MNAs in real time. The algorithm only takes $33.3 \mathrm{~ms}$ to compute TF features for a $4 \mathrm{~s}$ PPG window length using MATLAB running on a PC with the Intel Xeon processor operating at $3.6 \mathrm{GHz}$. Therefore, it would be straightforward to optimize the algorithm for real-time detection of MNAs in PPG data.

In conclusion, we propose an accurate MNA detection algorithm that utilizes both time and spectral features to classify between clean and corrupted PPG data segments. Moreover, it has the ability to go beyond MNA detection and can detect if the corrupted signal is still usable for accurate HR estimation or not. A comparison using four datasets showed our algorithm outperformed other contemporary MNA detection algorithms. Out algorithm also showed superiority with respect to detecting onset and conclusion of MNAs. Finally, TifMA is real-time realizable and it is applicable to either transmission (finger) or reflectance (forehead) recorded PPG signals.

\section{APPENDIX}

\section{VFCDM Features From PPG Signals}

VFCDM is a method for estimating TFS of a time-varying signal. This method was shown to provide concomitant high time and frequency resolution as well as preservation of the amplitude distribution of the signal [31]. VFCDM has two phases: 1) constructing an iTFS using a method developed in our laboratory, termed FFCDM; and 2) the center frequencies of the iTFS are used for further CDM to obtain even more accurate TFS and amplitude of the TFS. The VFCDM methodology is detailed as followed.

\section{VFCDM}

Consider a sinusoidal signal $x(t)$ to be a narrow band oscillation with a time-varying center frequency $f(\tau)$, instantaneous amplitude $A(t)$, phase $\phi(t)$, and the direct current component $\mathrm{dc}(t)$ :

$$
x(t)=\mathrm{dc}(t)+A(t) \cos \left(\int_{0}^{t} 2 \pi f(\tau)+f(t)\right) .
$$

For a given center frequency, we can extract the instantaneous amplitude information $A(t)$ and phase information $\phi(t)$ by mul- 
tiplying (1) by $\mathrm{e}^{-\int_{0}^{t} 2 \pi f(\tau) \mathrm{d} \tau}$ which results in the following:

$$
\begin{aligned}
z(t)= & x(t) \mathrm{e}^{-j \int_{0}^{t} 2 \pi f(\tau) \mathrm{d} \tau} \\
= & \mathrm{dc}(\mathrm{t}) \mathrm{e}^{-\mathrm{j} \int_{0}^{\mathrm{t}} 2 \pi \mathrm{f}(\tau) \mathrm{d} \tau}+\frac{\mathrm{A}(\mathrm{t})}{2} \mathrm{e}^{j f(t)} \\
& +\frac{A(t)}{2} \mathrm{e}^{-j\left(\int_{0}^{t} 4 \pi f(\tau)+f(t)\right)} .
\end{aligned}
$$

From (2), if $z(t)$ is filtered with an ideal low-pass filter (LPF) with a cutoff frequency $f_{c}<f_{0}$, where $f_{0}$ is the center frequency of interest. Then, the filtered signal $z_{\mathrm{lp}}(t)$ will contain only the component of interest:

$$
z_{\mathrm{lp}}(t)=\frac{A(t)}{2} \mathrm{e}^{j f(t)} .
$$

The instantaneous frequency is given by

$$
f(t)=f_{0}+\frac{1}{2 \pi} \frac{\mathrm{d} f(t)}{\mathrm{d} t} .
$$

By changing the center frequency followed by using the variable frequency approach as well as the LPF, the signal, $x(t)$, will be decomposed into the sinusoid modulations, $d_{i}$, by the CDM technique as follows:

$$
\begin{aligned}
x(t) & =\sum_{i} d_{i} \\
& =d c(t)+\sum_{i} A_{i}(t) \cos \left(\int_{0}^{t} 2 \pi f_{i}(\tau) \mathrm{d} \tau+f_{i}(t)\right) .
\end{aligned}
$$

The instantaneous frequency and amplitude of $d_{i}$ can be calculated using the Hilbert transform

$$
\begin{aligned}
& A(t)=2\left|z_{\mathrm{lp}}(t)\right|=\left[X^{2}(t)+Y^{2}(t)\right]^{1 / 2} \\
& X(t)=\operatorname{real}\left(z_{\mathrm{lp}}(t)\right)
\end{aligned}
$$

$$
\begin{aligned}
& Y(t)=\operatorname{imag}\left(z_{\mathrm{lp}}(t)\right)=\mathrm{H}[\mathrm{X}(\mathrm{t})]=\frac{1}{\pi} \int \frac{X\left(t^{\prime}\right)}{t-t^{\prime}} \mathrm{d} t^{\prime} \\
& \phi(t)=\arctan \left(\frac{\operatorname{imag}\left(z_{\mathrm{lp}}(t)\right)}{\operatorname{real}\left(z_{\mathrm{lp}}(t)\right)}\right)=\arctan \left(\frac{Y(t)}{X(t)}\right) .
\end{aligned}
$$

FFCDM operates by performing CDM on fixed frequency $f_{0}$ within confined bandwidth and repeat it over entire frequency band. In order to obtain even higher resolution TFS, center frequencies in iTFS obtained from FFCDM were used for subsequent CDM with finer bandwidth.

\section{REFERENCES}

[1] S. Dash, K. H. Chon, S. Lu, and E. A. Raeder, "Automatic real time detection of atrial fibrillation," Ann. Biomed. Eng., vol. 37, no. 9, pp. 17011709, Sep. 2009

[2] L. P. Antonsen and K. A. Kirkebøen, "Evaluation of fluid responsiveness: Is photoplethysmography a noninvasive alternative?" Anesthesiol. Res. Pract., vol. 2012, 2012, Art. no. 617380.

[3] F. Camacho, "Statistical analysis of central aortic blood pressure parameters derived from the peripheral pulse," Ph.D. dissertation, Biomed. Eng., Univ. New South Wales, Sydney, NSW, Australia, 2005.

[4] M. T. Petterson, V. L. Begnoche, and J. M. Graybeal, "The effect of motion on pulse oximetry and its clinical significance," Anesthesia Analgesia, vol. 105 , no. 6 Suppl, pp. S78-S84, Dec. 2007.
[5] R. Sahni, A. Gupta, K. Ohira-Kist, and T. Rosen, "Motion resistant pulse oximetry in neonates," Arch. Dis. Childhood Fetal Neonatal Ed., vol. 88, no. 6, pp. F505-F508, Nov. 2003.

[6] M. R. Ram, K. V. Madhav, E. H. Krishna, N. R. Komalla, and K. A. Reddy, "A novel approach for motion artifact reduction in PPG signals based on AS-LMS adaptive filter," IEEE Trans. Instrum. Meas., vol. 61, no. 5, pp. 1445-1457, May 2012.

[7] T. L. Rusch, R. Sankar, and J. E. Scharf, "Signal processing methods for pulse oximetry," Comput. Biol. Med., vol. 26, no. 2, pp. 143-159, Mar. 1996.

[8] R. Hong Enríquez, M. Sautié Castellanos, J. Falcón Rodríguez, and J. L. Hernández Cáceres, "Analysis of the photoplethysmographic signal by means of the decomposition in principal components," Physiol. Meas., vol. 23, no. 3, pp. N17-N29, Aug. 2002.

[9] S. M. A. Salehizadeh et al., "Photoplethysmograph signal reconstruction based on a novel hybrid motion artifact detection-reduction approach. Part II: Motion and noise artifact detection," Ann. Biomed. Eng., vol. 42, no. 11 , pp. 2251-2263, Nov. 2014.

[10] Y. Yan, C. C. Poon, and Y. Zhang, "Reduction of motion artifact in pulse oximetry by smoothed pseudo Wigner-Ville distribution," J. Neuroeng. Rehabil., vol. 2, pp. 1-9, Mar. 2005.

[11] J. Lee, W. Jung, I. Kang, Y. Kim, and G. Lee, "Design of filter to reject motion artifact of pulse oximetry," Comput. Stand. Interfaces, vol. 26, no. 3, pp. 241-249, May 2004

[12] K. V. P. Naraharisetti, M. Bawa, and M. Tahernezhadi, "Comparison of different signal processing methods for reducing artifacts from photoplethysmograph signal," in Proc. 2011 IEEE Int. Conf. Electro/Inf. Technol., 2011, pp. 1-8.

[13] M. R. Ram, K. V. Madhav, E. H. Krishna, K. N. Reddy, and K. A. Reddy, "Computation of $\mathrm{SpO} 2$ using non-parametric spectral estimation methods from wavelet based motion artifact reduced PPG signals," in Proc. 2011 Int. Conf. Signal Process. Commun. Comput. Netw. Technol., 2011, pp. 776-780.

[14] R. Krishnan, B. Natarajan, and S. Warren, "Two-stage approach for detection and reduction of motion artifacts in photoplethysmographic data," IEEE Trans. Biomed. Eng., vol. 57, no. 8, pp. 1867-1876,Aug. 2010.

[15] J. A. Sukor, S. J. Redmond, and N. H. Lovell, "Signal quality measures for pulse oximetry through waveform morphology analysis," Physiol. Meas., vol. 32, no. 3, pp. 369-384, Mar. 2011

[16] K. Li, S. Warren, and B. Natarajan, "Onboard tagging for real-time quality assessment of photoplethysmograms acquired by a wireless reflectance pulse oximeter," IEEE Trans. Biomed. Circuits Syst., vol. 6, no. 1, pp. 54 63, Feb. 2012.

[17] Q. Li, R. G. Mark, and G. D. Clifford, "Robust heart rate estimation from multiple asynchronous noisy sources using signal quality indices and a Kalman filter," Physiol. Meas., vol. 29, no. 1, pp. 15-32, Jan. 2008.

[18] K. Nakajima, T. Tamura, and H. Miike, "Monitoring of heart and respiratory rates by photoplethysmography using a digital filtering technique," Med. Eng. Phys., vol. 18, no. 5, pp. 365-372, Jul. 1996.

[19] W. Karlen, K. Kobayashi, J. M. Ansermino, and G. A. Dumont, "Photoplethysmogram signal quality estimation using repeated Gaussian filters and cross-correlation," Physiol. Meas., vol. 33, no. 10, pp. 1617-1629, Oct. 2012.

[20] B. Lee, J. Han, H. J. Baek, J. H. Shin, K. S. Park, and W. J. Yi, "Improved elimination of motion artifacts from a photoplethysmographic signal using a Kalman smoother with simultaneous accelerometry," Physiol. Meas., vol. 31, no. 12, pp. 1585-1603, Dec. 2010.

[21] J. Y. A. Foo and S. J. Wilson, "A computational system to optimise noise rejection in photoplethysmography signals during motion or poor perfusion states," Med. Biol. Eng. Comput., vol. 44, nos. 1/2, pp. 140-145, Mar. 2006.

[22] R. Krishnan, B. Natarajan, and S. Warren, "Analysis and detection of motion artifact in photoplethysmographic data using higher order statistics," in Proc. 2008 IEEE Int. Conf. Acoust., Speech, Signal Process., 2008, pp. 613-616.

[23] N. Selvaraj, Y. Mendelson, K. H. Shelley, D. G. Silverman, and K. H. Chon, "Statistical approach for the detection of motion/noise artifacts in photoplethysmogram," in Proc. Аnnu. Int. Conf. IEEE Eng. Med. Biol. Soc., vol. 2011, 2011, pp. 4972-4975.

[24] B. Hjorth, "The physical significance of time domain descriptors in EEG analysis," Electroencephalogr. Clin. Neurophysiol., vol. 34, no. 3, pp. 321325, Mar. 1973

[25] B. Hjorth, "EEG analysis based on time domain properties," Electroencephalogr. Clin. Neurophysiol., vol. 29, no. 3, pp. 306-310, Sep. 1970 
[26] E. Gil, J. María Vergara, and P. Laguna, "Detection of decreases in the amplitude fluctuation of pulse photoplethysmography signal as indication of obstructive sleep apnea syndrome in children," Biomed. Signal Process. Control, vol. 3, no. 3, pp. 267-277, Jul. 2008.

[27] J. W. Chong et al., "Photoplethysmograph signal reconstruction based on a novel hybrid motion artifact detection-reduction approach. Part I: Motion and noise artifact detection," Ann. Biomed. Eng., vol. 42, no. 11, pp. 2238-2250, Nov. 2014.

[28] C. G. Scully et al., "Using time-frequency analysis of the photoplethysmographic waveform to detect the withdrawal of $900 \mathrm{~mL}$ of blood," Anesthesia Analgesia, vol. 115, no. 1, pp. 74-81, Jul. 2012.

[29] N. Selvaraj, J. Lee, and K. H. Chon, "Time-varying methods for characterizing nonstationary dynamics of physiological systems," Methods Inf. Med., vol. 49, no. 5, pp. 435-442, 2010.

[30] N. Selvaraj et al., "A novel approach using time-frequency analysis of pulse-oximeter data to detect progressive hypovolemia in spontaneously breathing healthy subjects," IEEE Trans. Biomed. Eng., vol. 58, no. 8, pp. 2272-2279, Aug. 2011.
[31] H. Wang, K. Siu, K. Ju, and K. H. Chon, "A high resolution approach to estimating time-frequency spectra and their amplitudes," Ann. Biomed. Eng., vol. 34, no. 2, pp. 326-338, Feb. 2006.

[32] C. Hsu and C. Chang, A Practical Guide to Support Vector Classification. Taipei City, Taiwan: Dept. Comput. Sci., Nat. Taiwan Univ., 2003.

[33] M. Kearns and D. Ron, "Algorithmic stability and sanity-check bounds for leave-one-out cross-validation," Neural Comput., vol. 11, no. 6, pp. $1427-$ 1453, Aug. 1999.

Authors' photographs and biographies not available at the time of publication. 